\title{
The species distribution, antimicrobial resistance and risk factors for poor outcome of coagulase-negative staphylococci bacteraemia in China
}

\author{
Jiewei Cui ${ }^{1 \dagger}$, Zhixin Liang ${ }^{1 \dagger}$, Zhenfei Mo ${ }^{1}$ and Jianpeng Zhang ${ }^{2^{*}}$ (D)
}

\begin{abstract}
Objective: Coagulase-negative staphylococci (CoNS) are one of the major opportunistic pathogens and the incidence of CoNS bacteraemia is increasing. However, most of the CoNS-positive blood cultures are contaminants rather than true CoNS bacteraemia. In order to minimize contamination, we defined true CoNS bacteraemia as the patient that has two or more identical CoNS-positive blood cultures drawn within $48 \mathrm{~h}$ in this study and the objective of this study was to analyse the species distribution and antibiotic resistance and to identify risk factors for 30-day mortality of the true CoNS-bacteraemia.
\end{abstract}

Method: By reviewing the electronic medical database, this study retrospectively analysed patients diagnosed as CoNS bacteraemia by blood cultures in a comprehensive tertiary care hospital in China from January 1, 2014, to December 31, 2017.

Result: A total of 1241 patients with 1562 episodes of CoNS-positive blood cultures were recorded in the database but only 157 patients were finally diagnosed as true CoNS bacteraemia after contaminants were excluded. All these 157 patients $(12.7 \%, 157 / 1241)$ had bacteraemia-related clinical symptoms. Among the 157 patients, the most common species were Staphylococcus hominis (40.8\%), Staphylococcus epidermidis (36.3\%) and Staphylococcus capitis (11.5\%). The antimicrobial susceptibility tests showed that all CoNS had a high rate of resistance to penicillin (94.9\%) , oxacillin (93.6\%) and erythromycin (92.4\%). Resistance to gentamicin (22.3\%) and rifampicin (10.8\%) was low, and none of the bacteria were resistant to vancomycin or linezolid. The 30-day mortality of patients with CoNS bacteraemia was up to $12.7 \%$ (20/157), and the multivariate logistics regression analysis showed that chronic renal failure (OR 5.9, 95\% Cl 1.6-21.5, $p=0.007)$ and chronic liver failure $(\mathrm{OR} 4.0,95 \% \mathrm{Cl} 1.2-13.1, p=0.024)$ were both the significant independent risk factors for the 30-day mortality of CoNS bacteraemia.

Conclusion: Staphylococcus hominis and Staphylococcus epidermidis were the most common species in CoNS bacteraemia. All CoNS had high multi-drug resistance, but gentamicin and rifampicin had a relatively lower resistance and could be considered as alternative antibiotics for anti-CoNS bacteraemia in addition to vancomycin and linezolid. Additionally, patients with chronic renal failure or chronic liver failure have a higher 30-day mortality after the onset of CoNS bacteraemia.

Keywords: Coagulase-negative staphylococci, Bacteraemia, Species distribution, Antimicrobial resistance, Risk factors

\footnotetext{
* Correspondence: zjp99@vip.sina.com

†jiewei Cui and Zhixin Liang contributed equally to this work.

${ }^{2}$ Department of Respiratory Medicine, the Third Medical Centre of Chinese

PLA General Hospital, Yongding Road No.69, Beijing 100853, China

Full list of author information is available at the end of the article
}

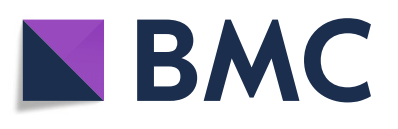

(c) The Author(s). 2019 Open Access This article is distributed under the terms of the Creative Commons Attribution 4.0 International License (http://creativecommons.org/licenses/by/4.0/), which permits unrestricted use, distribution, and reproduction in any medium, provided you give appropriate credit to the original author(s) and the source, provide a link to the Creative Commons license, and indicate if changes were made. The Creative Commons Public Domain Dedication waiver (http://creativecommons.org/publicdomain/zero/1.0/) applies to the data made available in this article, unless otherwise stated. 


\section{Background}

Coagulase-negative staphylococci (CoNS) are the most abundant common microbial species of the human skin and mucous membranes, and they are one of the major health care-associated opportunistic pathogens [1, 2]. CoNS can easily attach and grow on the surface of medical devices through a slime layer that has a muco-polysaccharide structure [3-5]. CoNS may cause bacteraemia when medical devices penetrate the skin and the mucous membrane barrier and enter the blood [6]. With the increasing use of invasive medical devices, such as the central venous catheter $(\mathrm{CVC})$ and periprosthetic joint $[7,8]$, the incidence of health care-associated CoNS bacteraemia is increasing [8-10].

CoNS are usually multi-drug resistant [11, 12]. The antimicrobial susceptibility of CoNS is critical for clinical empirical antibiotic therapy. Some recent studies suggested that CoNS were highly resistant to penicillin (PEN) (94.7\%), oxacillin (OXA) (90.7\%), and erythromycin (ERY) (85.3\%), and more than 50\% were resistant to cephalosporins, aminoglycosides and quinolones [13, 14]. However, most CoNS-positive blood cultures are false positives because CoNS could easily lead to blood culture contamination, and previous study suggested that the false-positive rates of CoNS-positive blood cultures were up to $90.9 \%$ if only one single CoNS-positive blood culture was present within $48 \mathrm{~h}$ [15]. According to false-positive blood cultures, antibiotics against CoNS may be overused, and patient care costs may be increased [16]. However, previous study had also pointed out that the false-positive rate dropped to $8.8 \%$ if an additional identical CoNS-positive blood culture was present within $48 \mathrm{~h}$ after the first CoNS-positive blood culture [15]. And up to now, there are rare studies on CoNS bacteraemia that had two or more identical CoNS-positive blood cultures drawn within $48 \mathrm{~h}$, although there were many studies on CoNS bacteraemia.

With the aim to determine the species distribution, antibiotic resistance and to identify risk factors for poor outcome of true CoNS-bacteraemia that had two or more identical CoNS-positive blood cultures drawn within $48 \mathrm{~h}$, we conducted the present study in a tertiary care hospital in China.

\section{Methods}

\section{Hospital setting}

This study was undertaken at the Chinese People's Liberation Army General Hospital (PLAGH), which is a 2200-bed, tertiary care hospital in Beijing, China. This centre is a comprehensive hospital with medical, health care, teaching, and scientific research accreditation that serves all military and nonmilitary personnel from all regions in China.

\section{Study design}

This was a retrospective study of hospitalized adult patients with CoNS-positive blood cultures in PLAGH from January 1, 2014, to December 31, 2017. All data were gathered by reviewing an ongoing prospective health care-associated infection surveillance electronic database of all positive blood cultures from all departments except paediatrics in the hospital.

\section{Case definitions}

The criteria used to classify CoNS as a pathogen and rule out contaminants in blood culture refer to a laboratory-based algorithm [15]. The patients with CoNS bacteraemia involved in the study met the inclusion criteria and the exclusion criteria as follows. Inclusion criteria: (1) The patients were aged $\geq 18$ years, patients under the age of 18 were excluded since risk factors and antimicrobial therapy for bloodstream infections among children are special. (2) The patients had at least two or more CoNS-positive blood cultures drawn within $48 \mathrm{~h}$, and the species were identical; however, only the first episode was included. (3) The patient must have had clinical symptoms or markers of inflammation associated with bacteraemia, e.g., fever $\left(>38^{\circ} \mathrm{C}\right)$, chills or elevated C-reactive protein (CRP). Exclusion criteria: (1) Patients who were pregnant or AIDS were excluded. (2) The onset of CoNS bacteraemia within the first $48 \mathrm{~h}$ after admission was excluded to ensure health care-associated infection [17]. (3) The patients with non-CoNS bacteraemia were excluded.

\section{Identification of isolates and antimicrobial susceptibility test}

Blood cultures were performed at the hospital's clinical microbiology laboratory. The blood culture was processed using the BacT/ALERT 3D Microbial Detection System (Becton-Dickinson, Sparks, MD, USA), and the species identification was performed using the VITEK 2 System (BioMérieux, Marcy 1'Étoile, France). Gram staining and coagulase testing were performed during the identification process. After the species identification, the antimicrobial susceptibility of isolated species was determined using standard procedures. Antibiotics for susceptibility: penicillin (PEN), oxacillin (OXA), erythromycin (ERY), cefoxitin (CEF), clindamycin (CLN), levofloxacin (LEV), moxifloxacin (MOX), trimethoprim/sulfamethoxazole (SUL), gentamicin (GEN), rifampicin (RIF), vancomycin (VAN) and linezolid (LIN). Methicillin resistance was determined by anti-OXA, which has a similar structure and antimicrobial susceptibility but better stability than PEN. The implementation standard of the antimicrobial susceptibility test was performed in accordance with the Clinical and Laboratory Standards Institute (CLSI) standards revised on January 1st, 2014 [18]. 


\section{Data collection}

The demographic and clinical characteristics were collected as follows: (1) Demographics: gender, age, body mass index (BMI), the onset date of CoNS bacteraemia (the date when the first CoNS-positive blood culture was drawn), total hospital stay length prior to the onset of CoNS bacteraemia, acute physiology and chronic health evaluation (APACHE) II scores and quick sepsis related organ failure assessment (qSOFA) scores which were evaluated based on medical records within $24 \mathrm{~h}$ after the onset of CoNS bacteraemia. (2) Treatments within 7 days prior to the onset of CoNS bacteraemia: indwelling CVC, surgery (including trauma), invasive mechanical ventilation, chemotherapy, renal replacement therapy including haemofiltration and dialysis, total parenteral nutrition, indwelling urinary catheter, glucocorticoid therapy (methylprednisolone $>1 \mathrm{mg} / \mathrm{kg} /$ day), and broad-spectrum antibiotics (defined as fourth-generation cephalosporins and carbapenems). (3) Comorbidities at the onset of CoNS bacteraemia: cardiovascular disease, pneumonia, cerebrovascular disease, diabetes mellitus, chronic renal failure, chronic liver failure, solid tumour, haematologic malignancy, and neutropenia. (4) Laboratory test results: inflammatory markers within $24 \mathrm{~h}$ after the onset of CoNS bacteraemia included the number of leukocytes, the number of neutrophils, and CRP. In addition, alanine aminotransferase (ALT) and serum creatinine (SCr) levels for assessing liver and renal dysfunction were also collected. (5) Antibiotics used for empirical antibiotic therapy within $72 \mathrm{~h}$ after the onset of CoNS bacteraemia. (6) Outcomes: survival or non-survival on the 30th day after the onset of CoNS bacteraemia was used to calculate 30-day mortality (all the data about outcomes not in the database were obtained by telephone follow-up).

The microbiological data collected included the isolated species of CoNS bacteraemia and their antimicrobial susceptibility results. According to the antimicrobial susceptibility results, empirical antibiotic therapy within $72 \mathrm{~h}$ after the onset of CoNS bacteraemia was considered appropriate if the antibiotic was sensitive. Additionally, empirical antibiotic therapy was also considered appropriate if the clinical symptom (temperature $>38^{\circ} \mathrm{C}$ ) recovered to normal (temperature $<38^{\circ} \mathrm{C}$ ) within $48 \mathrm{~h}$ after empirical antibiotic therapy.

\section{Statistical analysis}

Continuous variables were described as the mean (standard division $\mathrm{SD}$ ) or median (interquartile range, IQR), as appropriate according to normality. Categorical variables were described as frequency counts and percentages (n, $\%)$. In the analysis of risk factors for 30-day mortality, univariate regression analysis was used to initially screen for possible risk factors. All variables significant in univariate analysis were included in multivariate logistic and Cox regression analysis to analyse independent risk factors, but all the variables included in multivariate regression analysis should been performed statistical analysis on multicollinearity and correlation between risk factors to exclude the composite variables. All statistical analysis were performed using SPSS 22.0 software (IBM Corp., Armonk, NY, USA) and all results with a 2-tailed $p$-value $<0.05$ were considered significant.

\section{Results}

Details on patient enrolment

A total of 1241 adult patients with 1562 isolates of CoNS-positive blood cultures were identified in the ongoing prospective health care-associated infection surveillance electronic database from 2014 to 2017. Among them, only 163 patients had two or more CoNS-positive blood cultures with identical species within $48 \mathrm{~h}$. Four patients with no clinical symptoms or elevated inflammation markers were excluded, and one patient was excluded as a non-health care-associated infection since the onset of CoNS-positive blood culture was within the first $48 \mathrm{~h}$ after admission. In addition, one patient was excluded for having non-CoNS bacteraemia in addition to CoNS bacteraemia. Finally, 157 patients were diagnosed as health care-associated CoNS bacteraemia and were included in the study, which was $12.7 \%$ (157/1241) of all patients with CoNS-positive blood cultures. Among the 157 patients, the onset of the first CoNS bacteraemia was on January 9th, 2014. Details on patient enrolment were shown in Fig. 1.

\section{Microbiology and antimicrobial susceptibility}

The distribution and antimicrobial susceptibility of the species isolated among the 157 patients with CoNS bacteraemia are shown in Table 1. Among the 157 patients, the most common species was Staphylococcus hominis (64 isolates, 40.8\%), followed by Staphylococcus epidermidis (57 isolates, 36.3\%), Staphylococcus capitis (18 isolates, 11.5\%) and Staphylococcus haemolyticus (12 isolates, 7.6\%).

Overall, among all the species isolated, a high rate of resistance to OXA (93.6\%), PEN (94.9\%) and ERY (92.4\%) was observed, but no species were resistant to LIN and VAN. The resistance to methicillin was determined by OXA. Thus, the proportion of resistance to methicillin ranged from 83.3\% (Staphylococcus capitis) to 100\% (Staphylococcus haemolyticus), and methicillin-resistant Staphylococcus epidermidis (MRSE) accounted for 96.5\%. The antibiotic resistance details of each species are shown in Table 1.

\section{Demographic and clinical characteristics of the patients} The demographic and clinical characteristics of the 157 patients were shown in Table 2. The median age was 64 
1241 adult patients with 1562 isolates of CoNS-positive blood cultures were identified in the database. $(n=1241)$ $\longrightarrow \begin{aligned} & \text { Patients with only a single CoNS-postive blood } \\ & \text { culture with identical species within } 48 \mathrm{~h} \text { were } \\ & \text { excluded as being contaminated. }\end{aligned}$

163 patients had two or more CoNS-positive blood cultures with identical species within 48 h. $(n=163)$

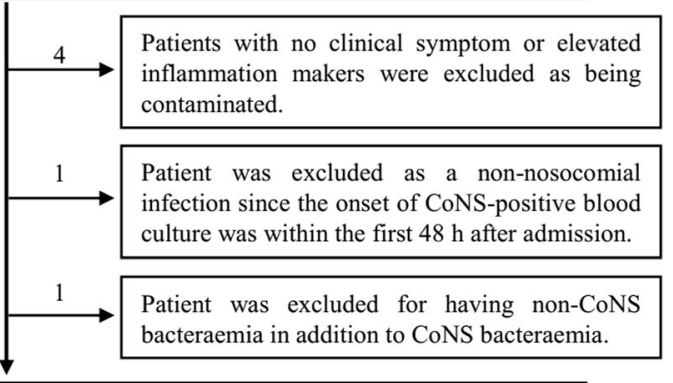

157 patients were finally diagnosed with nosocomial CoNS bacteraemia and only the first CoNS-positive blood culture of each patient was included in the study. $(n=157,12.7 \%)$

Fig. 1 Details on patient enrolment in this study

years (IQR, 48.0-80.0), age $\geq 60$ years accounted for 58.6\% (92/157), and 108 (68.8\%) patients were male.

At the onset of CoNS bacteraemia, $42.0 \%(66 / 157)$ of patients were residents in the ICU and the most common comorbidities were cardiovascular disease $(60.5 \%$, 95/157).

Prior to the onset of CoNS bacteraemia, the median length of total hospital stay was 15.0 days (IQR, 4.035.0 days), and the length $\geq 28$ days accounted for $33.8 \%$ (53/157). In addition, the most common treatments were indwelling CVC $(56.1 \%$ 88/157) within 7 days prior to the onset of CoNS bacteraemia.

Within $24 \mathrm{~h}$ after the onset of CoNS bacteraemia, the levels of ALT and SCr and the inflammatory markers including the number of leukocytes, number of neutrophils and the level of CRP exhibited in Table 2. Additionally, evaluated within this $24 \mathrm{~h}$ period, the APACHE II score and the qSOFA scores used to assess the severity of patients were also exhibited in Table 2.

Within $72 \mathrm{~h}$ after the onset of CoNS bacteraemia, empirical antibiotic therapy was appropriate among $132 \mathrm{pa}-$ tients $(84.1 \%, 132 / 157)$. VAN, which was used for empirical antibiotic therapy, accounted for $25.5 \%$ (40/ 157), carbapenems accounted for $25.5 \%$ (40/157), and LIN was only used among 4 patients.

\section{Outcomes and risk factors for poor outcome}

Most of our data regarding outcome were collected by reviewing the medical database, but there were 29 patients whose 30-day survival data were not available, because their hospital stay length did not reach 30 days from the onset of CoNS bacteraemia to discharge and we had followed up these 29 patients $(18.5 \%, 29 / 157)$ to collect the 30-day survival data by telephone. Our data showed that the 30-day mortality of the 157 patients with CoNS bacteraemia was $12.7 \%$ (20/157), and the median survival length from the onset of CoNS bacteraemia was 17.0 days (IQR, 7.8-21.2 days) among the 20 non-survival patients.

Univariate logistics analysis of risk factors associated with 30-day mortality of CoNS bacteraemia were also shown in Table 2. The results suggested that the 30-day mortality was possibly associated with age $\geq 60$ years (OR $3.2)$, residence in the ICU (OR 3.2), qSOFA scores (2 and 3) (OR 3.2), APACHE II score (OR 3.2), ALT (OR 3.2 ) and total hospital stay length prior to the onset of CoNS bacteraemia ( $\geq 28$ days) (OR 3.2). In addition, the comorbidities of pneumonia (OR 3.2), chronic renal failure (OR 3.2) and chronic liver failure (OR 3.2) were also associated risk factors. All the possible risk factors above were re-analysed in multivariate logistics analysis to find the independent risk factors. Additionally, although

Table 1 The distribution of species and their antimicrobial susceptibility in CoNS bacteraemia

\begin{tabular}{|c|c|c|c|c|c|c|c|c|c|c|c|c|c|}
\hline \multirow[t]{2}{*}{ Species } & \multirow{2}{*}{$\begin{array}{l}\text { No of } \\
\text { isolates } \\
(n, \%)\end{array}$} & \multicolumn{12}{|c|}{ Rate of resistance to a panel of 12 antibiotics (\%) } \\
\hline & & PEN & OXA & ERY & CEF & CLN & LEV & MOX & SUL & GEN & RIF & VAN & LIN \\
\hline Total & $157(100 \%)$ & 94.9 & 93.6 & 92.4 & 78.3 & 73.2 & 72.6 & 71.3 & 59.9 & 22.3 & 10.8 & 0 & 0 \\
\hline S. hominis & $64(40.8 \%)$ & 96.9 & 92.2 & 98.4 & 84.4 & 85.9 & 79.7 & 79.7 & 62.5 & 9.4 & 9.4 & 0 & 0 \\
\hline S. epidermidis & $57(36.3 \%)$ & 96.5 & 96.5 & 87.7 & 75.4 & 61.4 & 61.4 & 59.6 & 71.9 & 28.1 & 15.7 & 0 & 0 \\
\hline S. capitis & $18(11.5 \%)$ & 77.8 & 83.3 & 88.9 & 61.1 & 77.8 & 72.2 & 66.7 & 11.1 & 16.7 & 0 & 0 & 0 \\
\hline S. haemolyticus & $12(7.6 \%)$ & 100 & 100 & 91.7 & 91.7 & 50 & 100 & 100 & 66.7 & 75 & 16.7 & 0 & 0 \\
\hline S. saprophyticus ${ }^{a}$ & 3 & 3 & 3 & 3 & 1 & 2 & 0 & 0 & 1 & 0 & 0 & 0 & 0 \\
\hline S. cohnii ${ }^{a}$ & 2 & 2 & 2 & 1 & 2 & 2 & 2 & 2 & 2 & 0 & 0 & 0 & 0 \\
\hline S. sciuri ${ }^{a}$ & 1 & 1 & 1 & 1 & 1 & 1 & 1 & 1 & 0 & 1 & 0 & 0 & 0 \\
\hline
\end{tabular}

S. Staphylococcus, PEN penicillin, OXA oxacillin, ERY erythromycin, CEF cefoxitin, CLN clindamycin, LEV levofloxacin, MOX moxifloxacin, SUL trimethoprim/ sulfamethoxazole, GEN gentamicin, RIF rifampicin, VAN vancomycin and LIN linezolid

${ }^{a}$ The data were described as " $n$ " since each frequency was too small 
Table 2 Clinical Characteristics and univariate logistics analysis of risk factors for 30-day mortality of patients diagnosed with CoNS bacteraemia

\begin{tabular}{|c|c|c|c|c|c|c|}
\hline & $\begin{array}{l}\text { Total } \\
n=157\end{array}$ & $\begin{array}{l}\text { Survival } \\
n=137\end{array}$ & $\begin{array}{l}\text { Non-survival } \\
n=20\end{array}$ & OR & $95 \% \mathrm{Cl}$ & $p$-value \\
\hline$\overline{\text { Age }}$ & $64.0(48.0-80.0)$ & $62.0(48.0-80.0)$ & $72.0(62.8-80.2)$ & 1.0 & $1.0-1.0$ & 0.112 \\
\hline$\geq 60$ years & $92(58.6 \%)$ & $76(55.5 \%)$ & $16(80.0 \%)$ & 3.2 & $1.0-10.1$ & $0.046^{*}$ \\
\hline$<60$ years & $65(41.4 \%)$ & $61(44.5 \%)$ & $4(20.0 \%)$ & & & \\
\hline \multicolumn{7}{|l|}{ Gender } \\
\hline female & $49(31.2 \%)$ & $42(30.7 \%)$ & $7(35 \%)$ & 1.2 & $0.5-3.3$ & 0.696 \\
\hline male & $108(68.8 \%)$ & $95(69.3 \%)$ & $13(65.0 \%)$ & & & \\
\hline Residence in ICU & $66(42.0 \%)$ & $52(38.0 \%)$ & $14(70.0 \%)$ & 3.8 & $1.4-10.5$ & $0.010^{*}$ \\
\hline APACHE II score & $12.0(6.0-20.0)$ & $11.0(1.0-36.0)$ & $20.0(6.0-32.0)$ & 1.1 & $1.0-1.2$ & $0.002^{*}$ \\
\hline \multicolumn{7}{|l|}{ qSOFA score } \\
\hline 2 and 3 & $36(22.9 \%)$ & $26(19.0 \%)$ & $10(50.0 \%)$ & 4.3 & $1.6-11.3$ & $0.004^{*}$ \\
\hline 0 and 1 & $121(77.1 \%)$ & 111 (81.0\%) & $10(50.0 \%)$ & & & \\
\hline Prior hospital stay length & $15.0(4.0-35.0)$ & $13.0(4.0-31.0)$ & $30.5(5.8-46.2)$ & 2.8 & $1.0-1.0$ & 0.707 \\
\hline$\geq 28$ days & $53(33.8 \%)$ & $42(31 \%)$ & $11(55 \%)$ & 2.8 & $1.1-7.2$ & $0.036^{*}$ \\
\hline$<28$ days & $104(66.2 \%)$ & $95(69.3 \%)$ & $9(45.0 \%)$ & & & \\
\hline \multicolumn{7}{|l|}{ Comorbidities } \\
\hline Cardiovascular disease & $95(60.5 \%)$ & $82(59.9 \%)$ & $13(65.0 \%)$ & 1.2 & $0.5-3.3$ & 0.661 \\
\hline Pneumonia & $73(46.5 \%)$ & $59(43.1 \%)$ & $14(70.0 \%)$ & 3.1 & $1.1-8.5$ & $0.030^{*}$ \\
\hline Cerebrovascular disease & $46(29.3 \%)$ & $42(30.7 \%)$ & $4(20.0 \%)$ & 0.6 & $0.2-1.8$ & 0.333 \\
\hline Diabetes mellitus & $36(22.9 \%)$ & $32(23.4 \%)$ & $4(20.0 \%)$ & 0.8 & $0.3-2.6$ & 0.739 \\
\hline Chronic liver failure & $29(18.5 \%)$ & $20(14.6 \%)$ & $9(45.0 \%)$ & 4.8 & $1.8-13.0$ & $0.002^{*}$ \\
\hline Chronic renal failure & $27(17.2 \%)$ & $18(13.1 \%)$ & $9(45.0 \%)$ & 5.4 & $2.0-14.9$ & $0.001 *$ \\
\hline Solid tumor & $28(17.8 \%)$ & $24(17.5 \%)$ & $4(20.0 \%)$ & 1.2 & $0.4-3.8$ & 0.787 \\
\hline Hematologic malignancy & $11(7.0 \%)$ & $9(6.6 \%)$ & $2(10.0 \%)$ & 1.6 & $0.3-7.9$ & 0.577 \\
\hline Neutropenia & $12(7.6 \%)$ & $11(8.0 \%)$ & $1(5.0 \%)$ & 0.6 & $0.1-4.9$ & 0.637 \\
\hline \multicolumn{7}{|l|}{ Prior treatments } \\
\hline Indwelling CVC & $88(56.1 \%)$ & $74(54.0 \%)$ & $14(70.0 \%)$ & 2.0 & $0.7-5.5$ & 0.184 \\
\hline Indwelling urinary catheter & $67(42.7 \%)$ & $57(41.6 \%)$ & $10(50.0 \%)$ & 1.4 & $0.5-3.6$ & 0.480 \\
\hline Invasive MV & $38(24.2 \%)$ & $33(24.1 \%)$ & $5(25.0 \%)$ & 1.1 & $0.4-3.1$ & 0.929 \\
\hline Broad-spectrum antibiotics & $31(19.7 \%)$ & $45(32.8 \%)$ & $6(30.0 \%)$ & 0.9 & $0.3-2.4$ & 0.800 \\
\hline Surgery & $28(17.8 \%)$ & $26(19.0 \%)$ & $2(10.0 \%)$ & 0.5 & $0.1-2.2$ & 0.337 \\
\hline Renal replacement therapy & $9(5.7 \%)$ & $7(5.1 \%)$ & $2(10.0 \%)$ & 2.1 & $0.4-10.7$ & 0.389 \\
\hline Chemotherapy & $4(2.5 \%)$ & $3(2.2 \%)$ & $1(5.0 \%)$ & 2.4 & $0.2-23.8$ & 0.469 \\
\hline \multicolumn{7}{|l|}{ Laboratory test results } \\
\hline $\mathrm{CRP}(\mathrm{mg} / \mathrm{dL})$ & $3.9(2.3-8.6)$ & $3.9(0.1-29.4)$ & $3.9(1.2-78.2)$ & 1.0 & $1.0-1.1$ & 0.175 \\
\hline Leukocyte (X109/L) & $8.2(5.2-11.9)$ & $8.2(4.7-12.1)$ & $8.3(6.2-11.1)$ & 1.0 & $0.9-1.1$ & 0.706 \\
\hline Neutrophils (×109/L) & $6.5(3.6-9.5)$ & $6.6(3.3-9.9)$ & $6.3(4.1-9.1)$ & 1.0 & $0.9-1.1$ & 0.643 \\
\hline $\operatorname{ALT}(\mathrm{U} / \mathrm{L})$ & $24.6(13.5-41.9)$ & $24.4(3.7-147.0)$ & $30.2(1.7-419.1)$ & 1.0 & $1.0-1.0$ & $0.024 *$ \\
\hline $\mathrm{SCr}(\mu \mathrm{mol} / \mathrm{L})$ & $63.5(51.3-83.6)$ & $62.1(22.4-1392.0)$ & $69.0(37.9-791.0)$ & 1.0 & $1.0-1.0$ & 0.690 \\
\hline Appropriate empirical antibiotic therapy & $132(84.1 \%)$ & 117 (85.4\%) & $15(75.0 \%)$ & 0.5 & $0.2-1.6$ & 0.242 \\
\hline Vancomycin & $40(25.5 \%)$ & $39(28.5 \%)$ & $1(5.0 \%)$ & 0.1 & $0.0-1.0$ & 0.052 \\
\hline Carbapenems & $33(21.0 \%)$ & $27(19.7 \%)$ & $6(30.0 \%)$ & 1.7 & $0.6-5.0$ & 0.296 \\
\hline Linezolid & $4(2.5 \%)$ & 4 & 0 & - & - & - \\
\hline
\end{tabular}

OR odds ratio, $95 \% \mathrm{Cl}$ confidence interval, $C V C$ central venous catheter, $M V$ mechanical ventilation, $C R P$ c-reactive protein, $A L T$ alanine aminotransferase, $S C r$ serum creatinine, APACHE II acute physiology and chronic health evaluation (ii), qSOFA quick sepsis related organ failure assessment

*Bold font means that the risk factor was statistically $p<0.05$ in the univariate logistics analysis and was included in the multivariate logistics analysis 
appropriate empirical antibiotic therapy was not a statistically significant variable (OR $0.5,95 \%$ CI $0.2-1.6, p=$ 0.242 ) in our study, it was still included in the multivariate analysis since appropriate empirical antibiotic therapy was a clearly protective factor for outcome of infectious diseases in many previous studies. For example, appropriate antimicrobial therapy was the significant protective factor (OR $0.33, p=0.013$ ) in enterococcal bloodstream infections [19].

Before these variables above were included in the multivariate regression analysis, we performed collinearity and correlation analysis between these variables to exclude the composite variables. The multicollinearity results showed that the Tolerance of each variable was> 0.2 and the Variance Inflation Factor (VIF) of each variable was $<10$ and the largest VIF was 2.150 (APACHE II score), indicating that there was no multicollinearity between these risk factors. However, exhibited in Table 3, the correlation analysis showed that the spearman correlation coefficient ( $\mathrm{r}$ ) between the APACHE II score and the qSOFA score was big $(\mathrm{r}=0.594, p<0.05)$. In addition, shown in Table 3, the APACHE II score was also significantly correlated with other factors, such as age $(r=0.281)$, residence in ICU $(r=0.333)$, chronic renal failure $(r=0.282)$ and so on. Additionally, there are some same parameters used to calculate the value of the APACHE II score and the qSOFA score, and the qSOFA score is part of the APACHE II score. Hence, we believed that the APACHE II score might have multicollinearity with other factors and affect the statistical results. Therefore, we excluded the APACHE II score from the multivariate regression analysis. Similarly, as shown in Table 3, there was a significant correlation between ALT and chronic liver failure, although the correlation coefficient was small $(r=0.339, p<0.05)$. Additionally, taking into account the close relationship between chronic liver failure and the level of ALT in clinical practice, we thought they might be composite factors and we also ruled out the ALT from the multivariate regression analysis.

Finally, only 8 risk factors were included in the multivariate regression analysis (shown in Table 4). About sample size estimation in regression analysis, we had used the previous empirical method to assess whether our sample size was sufficient. In regression analysis, the sample size used for prognostic risk factor analysis requires at least 10 individuals for each prognostic factor. Because we included 8 risk factors in the multivariate regression analysis in our study (Table 4), the sample size required at least 80 patients. In our study, we included 157 patients and the sample size should theoretically be able to meet statistical needs. Because we did not perform power analysis to estimate the sample size before the statistical analysis, we could not report a post hoc power calculation.

At first, we had performed multivariate logistic regression analysis and the predicted values of the regression model was $91.7 \%$. The results suggested that chronic renal failure (OR 5.9, 95\% CI 1.6-21.5, $p=0.007$ ) and chronic liver failure (OR 4.0, 95\% CI 1.2-13.1, $p=0.024$ ) were both the significant independent risk factors for the 30-day mortality of CoNS bacteraemia in the regression model (Table 4).

Then, taking into account the influence of the time to event, we also performed multivariate Cox regression analysis. The Cox regression analysis showed that chronic renal failure (HR 3.9, 95\% CI 1.2-13.1, $p=$ 0.007 ) and chronic liver failure (HR 2.9, 95\% CI 1.1-7.6, $p=0.026)$ were still independent risk factor for the 30-day mortality of CoNS bacteraemia in the regression model (Table 4).

\section{Discussion}

CoNS bacteraemia is difficult to diagnose because the majority of CoNS-positive blood cultures are usually

Table 3 The correlation coefficient between factors planed to be included in the multivariate regression analysis model

\begin{tabular}{|c|c|c|c|c|c|c|c|c|c|}
\hline Correlation coefficient (r) & $\begin{array}{l}\text { APACHE } \\
\text { II score }\end{array}$ & ALT & $\begin{array}{l}\text { Age } \geq 60 \\
\text { years }\end{array}$ & $\begin{array}{l}\text { Residence } \\
\text { in ICU }\end{array}$ & $\begin{array}{l}\text { qSOFA } \\
(2 \text { and } 3)\end{array}$ & $\begin{array}{l}\text { Prior hospital stay } \\
\text { length ( } \geq 28 \text { days) }\end{array}$ & Pneumonia & $\begin{array}{l}\text { Chronic } \\
\text { liver failure }\end{array}$ & $\begin{array}{l}\text { Chronic } \\
\text { renal failure }\end{array}$ \\
\hline $\begin{array}{l}\text { Appropriate empirical } \\
\text { antibiotic therapy }\end{array}$ & -0.030 & 0.131 & 0.094 & -0.017 & 0.072 & -0.057 & -0.153 & 0.028 & -0.032 \\
\hline Chronic renal failure & $0.261 *$ & -0.007 & 0.006 & 0.125 & 0.073 & -0.111 & 0.049 & $0.218^{*}$ & 1.000 \\
\hline Chronic liver failure & $0.202^{*}$ & $0.339^{*}$ & 0.000 & 0.127 & 0.131 & 0.007 & 0.116 & 1.000 & / \\
\hline Pneumonia & $0.330^{*}$ & 0.055 & $0.265^{*}$ & $0.267^{*}$ & $0.251^{*}$ & $0.280^{*}$ & 1.000 & / & / \\
\hline Prior hospital stay length ( $\geq 28$ days) & $0.262^{*}$ & -0.109 & $0.217^{*}$ & $0.238^{*}$ & $0.251 *$ & 1.000 & / & / & / \\
\hline qSOFA score (2 and 3) & $0.594^{*}$ & 0.033 & 0.120 & $0.334^{*}$ & 1.000 & / & / & / & / \\
\hline Residence in ICU & $0.333^{*}$ & 0.067 & 0.061 & 1.000 & / & / & / & / & / \\
\hline Age ( $\geq 60$ years) & $0.281 *$ & -0.107 & 1.000 & / & / & / & / & / & / \\
\hline $\mathrm{ALT}(\mathrm{U} / \mathrm{L})$ & 0.033 & 1.000 & / & / & / & / & / & / & / \\
\hline
\end{tabular}

$A L T$ alanine aminotransferase

*Bold font means that correlation coefficient $(r)$ was statistically $p<0.05$ and there was a significant correlation between the two variables 
Table 4 The multivariate regression analysis of risk factors for 30-day mortality of patients diagnosed as CoNS bacteraemia

\begin{tabular}{|c|c|c|c|c|c|c|}
\hline & \multicolumn{3}{|c|}{ Logistics regression $^{a}$} & \multicolumn{3}{|c|}{ Cox regression } \\
\hline & $\overline{O R}$ & $95 \% \mathrm{Cl}$ & $p$-value & $\mathrm{HR}$ & $95 \% \mathrm{Cl}$ & $p$-value \\
\hline \multicolumn{7}{|l|}{ Age } \\
\hline$\geq 60$ years & 3.3 & $0.9-12.6$ & 0.084 & 2.6 & $0.8-8.4$ & 0.100 \\
\hline \multicolumn{7}{|l|}{$<60$ years } \\
\hline Residence in ICU & 1.7 & $0.5-5.6$ & 0.399 & 1.6 & $0.6-4.8$ & 0.371 \\
\hline \multicolumn{7}{|l|}{ qSOFA score } \\
\hline 2 and 3 & 2.8 & $0.9-9.4$ & 0.089 & 2.1 & $0.8-5.6$ & 0.143 \\
\hline \multicolumn{7}{|l|}{0 and 1} \\
\hline \multicolumn{7}{|l|}{ Prior hospital stay length } \\
\hline$\geq 28$ days & 2.6 & $0.8-9.0$ & 0.132 & 1.9 & $0.7-5.2$ & 0.184 \\
\hline \multicolumn{7}{|l|}{$<28$ days } \\
\hline Pneumonia & 1.2 & $0.4-4.1$ & 0.756 & 1.2 & $0.4-3.5$ & 0.687 \\
\hline Chronic liver failure & 4.0 & $1.2-13.1$ & $0.024^{*}$ & 2.9 & $1.1-7.6$ & $0.026^{*}$ \\
\hline Chronic renal failure & 5.9 & $1.6-21.5$ & $0.007^{*}$ & 3.9 & $1.5-10.5$ & $0.007^{*}$ \\
\hline Appropriate empirical antibiotic therapy & 0.3 & $0.1-1.1$ & 0.066 & 0.3 & $0.1-1.0$ & 0.060 \\
\hline
\end{tabular}

${ }^{a}$ The predicted values of the final model was $91.7 \%$. OR odds ratio, $H R$ hazard ratio, $C l$ confidence interval

*Bold font means that the risk factor was statistically $p<0.05$ and was the independent risk factor among the 8 factors

considered contamination or non-pathogens. The true rate of CoNS bacteraemia ranged from 5 to $39.6 \%$ [13, 20, 21]. In this study, a total of 1241 adult patients with CoNS-positive blood cultures were identified. However, the majority were excluded as contamination, only 157 (12.7\%) patients were finally confirmed as having CoNS bacteraemia, and their first CoNS-positive blood cultures were included in the study.

Among the 157 patients, the top two common species of CoNS were Staphylococcus hominis (40.8\%) and Staphylococcus epidermidis (36.3\%). This result was consistent with previous studies, although the proportion of Staphylococcus epidermidis demonstrated as the most common species of CoNS [4, 22]. Additionally, we found several rare species of CoNS, including three isolates of Staphylococcus saprophyticus, two isolates of Staphylococcus cohnii and 1 isolate of Staphylococcus sciuri.

Regarding CoNS resistance, CoNS bacteraemia species showed a multi-drug resistance profile in many previous studies [11, 23, 24]. In our study, 12 antibiotics were tested for antimicrobial susceptibility. As shown in Table 1 , among the 157 patients, resistance to penicillin (94.9\%), oxacillin $(93.6 \%)$ and erythromycin $(92.4 \%)$ was found in almost all species of CoNS, and the three antibiotics could not be recommended for empirical antibiotic therapy of CoNS bacteraemia. The resistance to cefoxitin (78.3\%), clindamycin (73.2\%), levofloxacin (72.6\%) and trimethoprim/sulfamethoxazole (59.9\%) were slightly lower but still very high, and these antibiotics must be cautiously selected for anti-CoNS bacteraemia. OXA was used as the indication for methicillin resistance; thus, $93.6 \%$ of the 157 species of CoNS were considered methicillin resistant, and $96.5 \%$ of Staphylococcus epidermidis were able to be defined as MRSE. This result was similar to research data in southwestern Saudi Arabia [13] and other countries [23, 25]. Not surprisingly, consistent with most previous studies, resistance to vancomycin or linezolid was not found $[4,26]$. Multi-drug resistance has made vancomycin the first choice for anti-CoNS, and 25.5\% (40/157) of patients were prescribed VAN for empirical antibiotic therapy within $72 \mathrm{~h}$ after the onset of CoNS bacteraemia in this study (Table 2). However, VAN-resistant CoNS does exist, for example, Anne Santerre Henriksen et al. reported that 1 out of 460 isolates displayed resistance to VAN in their study [27]. For this reason, although VAN-resistant CoNS was not present in our study, we still inferred that VAN-resistant CoNS bacteraemia may appear, and it deserves our attention in the clinic. In addition to the above, we were surprised to find that CoNS resistance to gentamicin (22.3\%) and rifampicin $(10.8 \%)$ was relatively low, and these antibiotics could be considered as alternative drugs for clinical anti-CoNS treatment in some proper patients, in addition to vancomycin and linezolid.

In addition to species distribution and antimicrobial resistance, we also analysed the risk factors for poor outcome among the 157 patients in this study. As shown in Table 2, our results suggested that patients aged $\geq 60$ years (58.6\%) and males (68.8\%) accounted for the majority. The 30-day mortality of patients with CoNS bacteraemia was up to $12.7 \%(20 / 157)$. We observed in univariate 
analysis that the 30-day mortality was possibly associated with 9 possible risk factors such age $\geq 60$ years (Table 2). After ruled out the possible composite factors by the correlation and multicollinearity analysis (Table 3), we selected 8 factors to be included in the multivariate regression analysis (Table 4). The multivariate logistics regression analysis suggested that chronic renal failure and chronic liver failure were statistically significant independent risk factors, and the results were verified by multivariate Cox regression analysis (Table 4). Thus, the results indicated that the 30-day mortality of patients with chronic renal failure was 5.9 times than that of patients without chronic renal failure (OR 5.9, 95\% CI 1.6-21.5, $p=0.007$ ) after the onset of CoNS bacteraemia. Similarly, the 30-day mortality of patients with chronic liver failure was 4.0 times than that of patients without chronic liver failure (OR 4.0, 95\% CI 1.2-13.1, $p=0.024$ ).

The results were understandable and acceptable, since a large number of studies on chronic renal failure had suggested that infection was a clear risk factor for acute exacerbation and death in chronic renal failure. For example, the high rate of infectious complication was found to be a major determinant of 2-year mortality after coronary artery bypass grafting in patients with chronic renal disease (HR 4.42-9.39) [28]. Conversely, chronic renal failure was also a risk factor for 30-day mortality in patients with catheter-related bloodstream infection (OR 11.1) [29] and in patients with Pseudomonas aeruginosa bacteremia (OR 4.93) [30].

Similarly, infections was also one of the most common precipitating factors in acute-on-chronic liver failure [31]. For example, Junjun Cai et al. found Infections (regardless of first or second infection) can increase the 90-day mortality significantly in patients with acute-on-chronic liver failure and the presence of second infection were independent risk factors (OR 2.37) for 90-day mortality in acute-on-chronic liver failure after the first infection [32]. On the other hand, chronic liver failure was also a risk factor for death in bacteraemia. For example, chronic liver failure was an independent risk factors (OR 3.3) associated with 30-day mortality in enterococcal bacteraemia [19].

Our results had also exhibited similar conclusions in our study. We believed that the onset of CoNS bacteraemia could promote the aggravation of chronic renal failure (or chronic liver failure), and conversely, the risk of 30-day mortality of patients with chronic renal failure (or chronic liver failure) was inevitably higher than that of patients without chronic renal failure (or chronic liver failure) after the onset of CoNS bacteraemia. From these perspectives, our data results were just the reflection of these clinical phenomena rather than accidental results.

In addition to chronic renal failure and chronic liver failure, our results also showed that age $\geq 60$ years (OR
3.3, $p=0.084$ ), residence in ICU (OR 1.7, $p=0.399$ ), qSOFA (2 and 3) (OR 2.8, $p=0.089$ ) and pior hospital stay length $\geq 28$ days (OR 2.6, $p=0.132$ ) were also the risk factors for 30-day mortality, and appropriate empirical antibiotic therapy was the protective factor (OR 0.3, $p=0.066$ ). They were statistically significant independent risk factors or protective factors in some previous studies of infectious diseases although these factors were not significant in our study. The reason for the lack of significance of these factors might be that the sample size was still not sufficient in our study.

In conclusion, we analysed the species distribution, antibiotic resistance and risk factors for 30-day mortality in 157 patients with CoNS bacteraemia. We found that most species were Staphylococcus hominis and Staphylococcus epidermidis. All CoNS had high antibiotic resistance, 93.6\% were resistant to methicillin, and none was resistant to vancomycin or linezolid. Additionally, our results had also demonstrated that chronic renal failure and chronic liver failure were the independent risk factors for 30-day mortality of CoNS bacteraemia. Patients with chronic renal failure or chronic liver failure have a higher 30-day mortality after the onset of CoNS bacteraemia and their treatments should be attracted more attention to prevent and reduce the occurrence of death as much as possible.

\section{Limitations}

Several limitations also existed in the study. First, this study was retrospective and observational, and some unmeasured confounding factors might exist. For example, antimicrobial susceptibility to carbapenems was not tested according to the 2014 CLSI standards, but 32 out of 157 patients (21\%) were given carbapenems for empirical antibiotic therapy, and their effectiveness could be assessed only through clinical symptoms rather than drug susceptibility testing. This might have produced small errors, but we consider it to have little effect on the analysis of risk factors for mortality. Second, the number of patients was limited in this study in a single centre. Although 1241 patients had CoNS-positive blood cultures, only 157 patients were included in the study, and other CoNS-positive blood cultures were mainly considered as being contaminated. However, we found that some mild patients with true CoNS bacteraemia might recover quickly after the first CoNS-positive blood culture, but no additional blood culture was drawn. Therefore, some mild patients with true CoNS bacteraemia may be mistakenly excluded from the study. This may lead to overestimation of the severity and the 30-day mortality of CoNS bacteraemia. However, this is clinically acceptable because it helps reduce medical expenses and overuse of antibiotics by reducing excessive attention to patients who are mild and recover quickly. 


\section{Abbreviations}

95\% Cl: 95\% confidence interval; ALT: Alanine aminotransferase; APACHE II: Acute physiology and chronic health evaluation (ii); CEF: Cefoxitin; CLN: Clindamycin; CLSI: Clinical and Laboratory Standards Institute; CoNS: Coagulase-negative staphylococci; CRP: C-reactive protein; CVC: Central venous catheter; ERY: Erythromycin; GEN: Gentamicin; IQR: Interquartile range; LEV: Levofloxacin; LIN: Linezolid; MOX: Moxifloxacin; MRSE: Methicillin-resistant Staphylococcus epidermidis; OR: Odds ratio; OXA: Oxacillin; PEN: Penicillin; PLAGH: Chinese People's Liberation Army General Hospital; qSOFA: Quick sepsis related organ failure assessment; RIF: Rifampicin; SCr: Serum creatinine; SUL: Trimethoprim/sulfamethoxazole; VAN: Vancomycin

\section{Acknowledgements}

We thank all of our colleagues working on the ongoing prospective health care-associated infection surveillance electronic database in the PLAGH hospital. Without their prior efforts, this study could not be possible.

\section{Funding}

This study was supported by the National Key R\&D Program of China (Grant number 2016YFC1304700)

\section{Availability of data and materials}

The data generated or analysed during this study are not publicly available to avoid violating the privacy rules of the medical database in our hospital, but all data are available from the corresponding author for any reader if necessary.

\section{Authors' contributions}

JZ conducted the design of this study and was responsible for the manuscript. JC and ZL collected the clinical data, analysed the results and wrote the manuscript. ZM participated in data collection of the manuscript. All authors read and approved the final manuscript.

\section{Ethics approval and consent to participate}

The Clinical Trial Ethics Review Committee of the PLAGH Hospital approved this study. Because this study was a retrospective study and private information, such as patient name and address, was not involved in the study, formal consent was waived in our hospital.

\section{Consent for publication}

Not applicable.

\section{Competing interests}

The authors declare that they have no competing interests.

\section{Publisher's Note}

Springer Nature remains neutral with regard to jurisdictional claims in published maps and institutional affiliations.

\section{Author details}

'Department of Respiratory Medicine, the First Medical Centre of Chinese PLA General Hospital, Fuxing Road No. 28, Beijing 100853, China. ${ }^{2}$ Department of Respiratory Medicine, the Third Medical Centre of Chinese PLA General Hospital, Yongding Road No.69, Beijing 100853, China.

\section{Received: 18 January 2019 Accepted: 12 April 2019}

\section{Published online: 24 April 2019}

\section{References}

1. Ahlstrand E, Persson L, Tidefelt U, Soderquist B. Alteration of the colonization pattern of coagulase-negative staphylococci in patients undergoing treatment for hematological malignancy. Eur J Clin Microbiol Infect Dis. 2012;31(7):1679-87.

2. Piette A, Verschraegen G. Role of coagulase-negative staphylococci in human disease. Vet Microbiol. 2009;134(1-2):45-54.

3. Becker K, Heilmann C, Peters G. Coagulase-negative staphylococci. Clin Microbiol Rev. 2014;27(4):870-926.

4. Shrestha LB, Bhattarai NR, Khanal B. Antibiotic resistance and biofilm formation among coagulase-negative staphylococci isolated from clinical samples at a tertiary care hospital of eastern Nepal. Antimicrob Resist Infect Control. 2017;6:89.
5. Rampelotto RF, Lorenzoni W, Silva DDC, Coelho SS, Wust V, Garzon LR, et al. Assessment of different methods for the detection of biofilm production in coagulase-negative staphylococci isolated from blood cultures of newborns. Rev Soc Bras Med Trop. 2018:51(6):761-7.

6. Sabate Bresco M, Harris LG, Thompson K, Stanic B, Morgenstern M, O'Mahony $\mathrm{L}$, et al. Pathogenic mechanisms and host interactions in Staphylococcus epidermidis device-related infection. Front Microbiol. 2017:8:1401.

7. Gao Z, Du Y, Piao S, Sun J, Li X, Zhou Y. Comparison between the staphylococci aureus and coagulase-negative staphylococci infected total joint arthroplasty treated by two-stage revision: a retrospective study with two year minimum follow-up. J Orthop Sci. 2019;24(1):109-15.

8. Lourtet-Hascoet J, Felice MP, Bicart-See A, Bouige A, Giordano G, Bonnet E. Species and antimicrobial susceptibility testing of coagulase-negative staphylococci in periprosthetic joint infections. Epidemiol Infect. 2018; 146(14):1771-6.

9. Spelman T, Pilcher DV, Cheng AC, Bull AL, Richards MJ, Worth L. Central line-associated bloodstream infections in Australian ICUs: evaluating modifiable and non-modifiable risks in Victorian healthcare facilities. Epidemiol Infect. 2017;145(14):3047-55.

10. Zhu Q, Yue Y, Zhu L, Cui J, Zhu M, Chen L, et al. Epidemiology and microbiology of gram-positive bloodstream infections in a tertiary-care hospital in Beijing, China: a 6-year retrospective study. Antimicrob Resist Infect Control. 2018;7:107.

11. Singh S, Dhawan B, Kapil A, Kabra SK, Suri A, Sreenivas V, et al. Coagulasenegative staphylococci causing blood stream infection at an Indian tertiary care hospital: prevalence, antimicrobial resistance and molecular characterisation. Indian J Med Microbiol. 2016;34(4):500-5.

12. Xu Z, Shah HN, Misra R, Chen J, Zhang W, Liu Y, et al. The prevalence, antibiotic resistance and mecA characterization of coagulase negative staphylococci recovered from non-healthcare settings in London, UK. Antimicrob Resist Infect Control. 2018;7:73.

13. Asaad AM, Ansar Qureshi M, Mujeeb Hasan S. Clinical significance of coagulase-negative staphylococci isolates from nosocomial bloodstream infections. Infect Dis (Lond). 2016:48(5):356-60.

14. Shah MU, Akram MF, Usman J, Kaleem F. Incidence and susceptibility pattern of methicillin resistant coagulase-negative staphylococci isolated from a tertiary care hospital of Pakistan. Jundishapur J Microbiol. 2014;7(1):e8590.

15. Richter SS, Beekmann SE, Croco JL, Diekema DJ, Koontz FP, Pfaller MA, et al. Minimizing the workup of blood culture contaminants: implementation and evaluation of a laboratory-based algorithm. J Clin Microbiol. 2002;40(7): 2437-44.

16. Molina J, Penuela I, Lepe JA, Gutierrez-Pizarraya A, Gomez MJ, Garcia-Cabrera E, et al. Mortality and hospital stay related to coagulase-negative staphylococci bacteremia in non-critical patients. J Inf Secur. 2013;66(2):155-62.

17. Bastiaens GJH, Cremers AJH, Coolen JPM, Nillesen MT, Boeree MJ, Hopman $J$, et al. Nosocomial outbreak of multi-resistant Streptococcus pneumoniae serotype 15A in a Centre for chronic pulmonary diseases. Antimicrob Resist Infect Control. 2018;7:158.

18. CLSI. Performance Standards for Antimicrobial Susceptibility Testing: Twenty-Fourth Informational Supplement. CLSI document M100-S24. Wayne, PA: Clinical and Laboratory Standards Institute; 2014.

19. Suppli M, Aabenhus R, Harboe ZB, Andersen LP, Tvede M, Jensen JU. Mortality in enterococcal bloodstream infections increases with inappropriate antimicrobial therapy. Clin Microbiol Infect. 2011;17(7):1078-83.

20. Olaechea PM, Alvarez-Lerma F, Palomar M, Insausti J, Lopez-Pueyo MJ, Martinez-Pellus A, et al. Impact of primary and intravascular catheter-related bacteremia due to coagulase-negative staphylococci in critically ill patients. Med Int. 2011;35(4):217-25.

21. Tashiro M, Izumikawa K, Ashizawa N, Narukawa M, Yamamoto Y. Clinical significance of methicillin-resistant coagulase-negative staphylococci obtained from sterile specimens. Diagn Microbiol Infect Dis. 2015;81(1):71-5.

22. Bhatt $P$, Tandel $K$, Singh A, Mugunthan M, Grover N, Sahni AK. Species distribution and antimicrobial resistance pattern of coagulase-negative staphylococci at a tertiary care Centre. Med J Armed Forces India. 2016;72(1):71-4.

23. Pedroso S, Sandes SHC, Filho RAT, Nunes AC, Serufo JC, Farias LM, et al. Coagulase-negative staphylococci isolated from human bloodstream infections showed multidrug resistance profile. Microb Drug Resist. 2018; 24(5):635-47.

24. Deyno S, Fekadu S, Seyfe S. Prevalence and antimicrobial resistance of coagulase negative staphylococci clinical isolates from Ethiopia: a metaanalysis. BMC Microbiol. 2018;18(1):43. 
25. Rasigade JP, Raulin O, Picaud JC, Tellini C, Bes M, Grando J, et al. Methicillinresistant Staphylococcus capitis with reduced vancomycin susceptibility causes late-onset sepsis in intensive care neonates. PLoS One. 2012;7(2): e31548.

26. Bhatt P, Tandel K, Singh A, Kumar M, Grover N, Sahni AK. Prevalence and molecular characterization of methicillin resistance among coagulasenegative staphylococci at a tertiary care center. Med J Armed Forces India. 2016;72(Suppl 1):S54-S8.

27. Henriksen AS, Smart J, Hamed K. Comparative activity of ceftobiprole against coagulase-negative staphylococci from the BSAC Bacteraemia surveillance Programme, 2013-2015. Eur J Clin Microbiol Infect Dis. 2018;37: 1653-9.

28. Akman B, Bilgic A, Sasak G, Sezer S, Sezgin A, Arat Z, et al. Mortality risk factors in chronic renal failure patients after coronary artery bypass grafting. Ren Fail. 2007:29(7):823-8.

29. Lee YM, Moon C, Kim YJ, Lee HJ, Lee MS, Park KH. Clinical impact of delayed catheter removal for patients with central-venous-catheter-related gram-negative bacteraemia. J Hosp Infect. 2018;99(1):106-13.

30. Tuon FF, Gortz LW, Rocha JL. Risk factors for pan-resistant Pseudomonas aeruginosa bacteremia and the adequacy of antibiotic therapy. Braz I Infect Dis. 2012 Jul-Aug;16(4):351-6.

31. Solé C, Solà E. Update on acute-on-chronic liver failure. Gastroenterol Hepatol. 2018;41(1):43-53.

32. Cai J, Zhang M, Han T, Jiang HQ. Characteristics of infection and its impact on short-term outcome in patients with acute-on-chronic liver failure. Medicine (Baltimore). 2017;96(37):e8057.

Ready to submit your research? Choose BMC and benefit from:

- fast, convenient online submission

- thorough peer review by experienced researchers in your field

- rapid publication on acceptance

- support for research data, including large and complex data types

- gold Open Access which fosters wider collaboration and increased citations

- maximum visibility for your research: over $100 \mathrm{M}$ website views per year

At $\mathrm{BMC}$, research is always in progress.

Learn more biomedcentral.com/submissions 